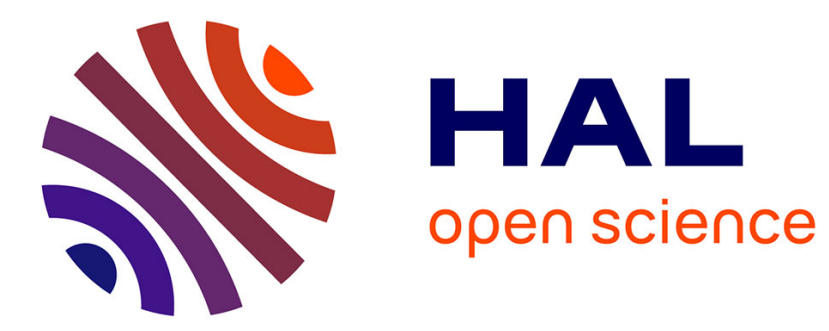

\title{
Practical Semantic Analysis of Web Sites and Documents
}

Thierry Despeyroux

\section{To cite this version:}

Thierry Despeyroux. Practical Semantic Analysis of Web Sites and Documents. 13th World Wide Web Conference, International World Wide Web Conference Committee and Association for Computing Machinery, May 2004, New York City/USA. inria-00000421

\section{HAL Id: inria-00000421 https://hal.inria.fr/inria-00000421}

Submitted on 11 Oct 2005

HAL is a multi-disciplinary open access archive for the deposit and dissemination of scientific research documents, whether they are published or not. The documents may come from teaching and research institutions in France or abroad, or from public or private research centers.
L'archive ouverte pluridisciplinaire HAL, est destinée au dépôt et à la diffusion de documents scientifiques de niveau recherche, publiés ou non, émanant des établissements d'enseignement et de recherche français ou étrangers, des laboratoires publics ou privés. 


\title{
Practical Semantic Analysis of Web Sites and Documents
}

\author{
Thierry Despeyroux \\ I.N.R.I.A. - Rocquencourt \\ AxIS Group \\ B.P. 105 - 78153 Le Chesnay Cedex, France \\ thierry.despeyroux@inria.fr
}

\begin{abstract}
As Web sites are now ordinary products, it is necessary to explicit the notion of quality of a Web site. The quality of a site may be linked to the easiness of accessibility and also to other criteria such as the fact that the site is up to date and coherent. This last quality is difficult to insure because sites may be updated very frequently, may have many authors, may be partially generated and in this context proof-reading is very difficult. The same piece of information may be found in different occurrences, but also in data or metadata, leading to the need for consistency checking.

In this paper we make a parallel between programs and Web sites. We present some examples of semantic constraints that one would like to specify (constraints between the meaning of categories and sub-categories in a thematic directory, consistency between the organization chart and the rest of the site in an academic site). We present quickly the Natural Semantics [12, 印, a way to specify the semantics of programming languages that inspires our works. Then we propose a specification language for semantic constraints in Web sites that, in conjunction with the well known "make" program, permits to generate some site verification tools by compiling the specification into Prolog code. We apply our method to a large XML document which is the scientific part of our institute activity report, tracking errors or inconsistencies and also constructing some indicators that can be used by the management of the institute.
\end{abstract}

\section{Categories and Subject Descriptors}

D.1.6 [Software]: Programming TechniquesLogic Programming; H.3.m [Information Systems]: Miscellaneous; I.7.2 [Computing Methodologies]: Document and Text ProcessingDocument Preparation[Languages and systems,Markup Languages]

\section{General Terms}

Experimentation, Verification

\section{Keywords}

consistency, formal semantics, logic programming, web sites, information system, knowledge management, content management, quality, XML, Web site evolution, Web engineering

\section{INTRODUCTION}

Web sites can be seen as a new kind of everyday product which is subject to a complex cycle of life: many authors, frequent updates

Copyright is held by the author/owner(s).

WWW2004, May 17-22, 2004, New York, New York, USA.

ACM 1-58113-844-X/04/0005. or redesigns. As for other "industrial" products, one can think of the notion of quality of a Web site, and then look for methods to achieve this quality.

Until now, the main effort developed in the domain of the Web is called the Semantic Web [1, 3]. Its goal is to ease a computer based data mining, formalizing data which is most of the time textual. This leads to two directions:

- Giving a syntactical structure to documents. This is achieved with XML, DTDs, XML-schema, style sheets and XSLT [20]. The goal is to separate the content of Web pages from their visual appearance, defining an abstract syntax to constrain the information structure. In this area, there is, of course, the work done by the $\mathrm{W} 3 \mathrm{C}$, but we can also mention tools that manipulate XML documents, taking into account the conformance to DTD: Xduce [11] and XM- $\lambda$ [13]. This means that by using these languages we know that the documents which are produced will conform to the specific DTDs that they use, which is not the case when one uses XSLT.

- Annotating documents to help computerized Web mining. One will use ontologies with the help of RDF [20, 2], RDFSchema or DAML+OIL [21]. The goal is to get a computer dedicated presentation of knowledge [18, 8, 7, to check the content of a page with the use of ontologies [19] or to improve information retrieval.

Our approach is different as we are concerned in the way Web sites are constructed, taking into account their development and their semantics. In this respect we are closer to what is called content management. To do that, we use some techniques coming from the world of the semantics of programming languages and from software engineering.

Web sites, as many other types of information systems, contain naturally redundant information. This is in part due to accessibility reasons. Web pages can be annotated and the same piece of information can exist in many different forms, as data or meta-data. We have to make sure that these different representations of the same piece of knowledge are consistent. Some parts of a Web site can also be generated, from one or more databases, and again, one have to make sure of the consistency between these databases and the rest of the site.

Obviously, traditional proof-reading (as it may be done for a book) is not possible for Web sites. A book may contain a structure (chapters, sections, etc.) and references across the text, but its reading can be essentially linear. An information system such as a Web site looks more like a net.

We propose to apply techniques from software engineering to increase the quality level of Web sites. In the context of Web sites, it is not possible to make proofs as it is the case for some sorts of 
programs or algorithms, but we will see that some techniques used in the area of formal semantics of programming languages [10] can be successfully used in this context.

The work presented in this paper is limited to static Web sites and documents. It can be easily extended to more general information systems as many of them provide a Web interface or at least can generate XML documents, answering to the two questions that we try to solve: How can we define verification tools for Web sites an more generally information systems? How can we mechanize the use of these tools?

In a first section we will make a parallel between programs and Web sites. In the second one we will show some examples of semantics constraints in Web sites. Then we will explore the Natural Semantics approach and will see how to adapt this method to our current problem. We will finish this paper by describing experiments that have been done and some implementation notes.

\section{FROM PROGRAMS TO WEB SITES}

To execute a program you have, most of the time, to use a compiler which translates the source code to executable code, unless you are the end-user and someone else did this for you.

The goal of a compiler is not only to generate object code but also to verify that the program is legal, i.e., that it follows the static semantics of the programming language. For example, in many programming languages one can find some declarative parts in which objects, types and procedures are defined to be used in some other places in statements or expressions. One will have to check that the use of these objects is compatible with the declarations.

The static semantics is defined by opposition to the dynamic semantics which express the way a program is executed. The static semantics express some constraints that must be verified before a program is executed or compiled.

A particularity of such constraints is that they are not local but global: they may bind distant occurrences of an object in a unique file or in many different files. A second particularity is that these constraints are not context-independent: an "environment" that allows us to know what are the visible objects at a particular point in the program is necessary to perform the verifications.

Global constraints are defined by opposition to local constraints. As a program may be represented by a labeled tree, a local constraint is a relation between a node in this tree and its sons. For example, if we represent an assignment, the fact that the right hand part of an assignment must be an expression is a local constraint. On the other hand, the fact that the two sides of an assignment must have compatible types is a global constraint, as we need to compute the types of both sides using an environment.

Local constraints express what is called the (abstract) syntax of the programming language and global constraints express what is called its static semantics. The abstract syntax refers to the tree representation of a program, its textual form is called its concrete syntax.

Representing programs by means of a tree is quite natural. Using a B.N.F. to describe the syntax of a programming language gives already a parse tree. Most of the time this tree can be simplified to remove meaningless reduction level due to precedence of operators. The grammar rules express local type constraints on the parse tree and explain what is a syntacticly correct program. A straight way of representing a program in a language like Prolog is to use (completely instantiated, i.e., with no logical variables) terms, even if Prolog terms are not typed.

The following example shows how a statement can be represented as a Prolog term.

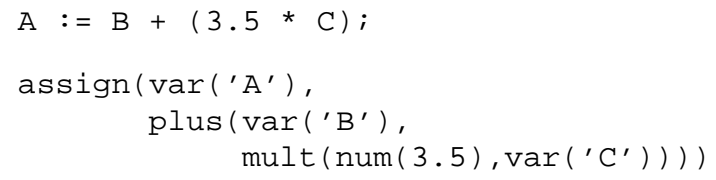

A Prolog term can itself be represented into XML as shown in the following example:

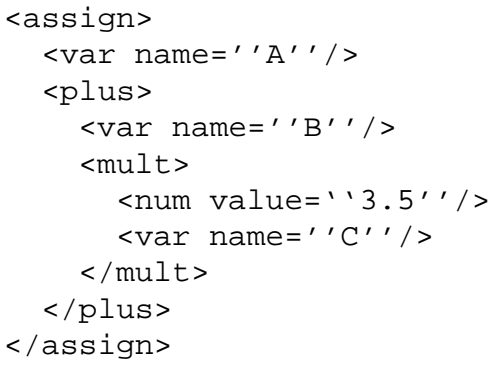

By defining a DTD for our language, we can constrain XML to allow only programs that respect the syntax of the programming language.

<!ELEMENT assign (var, (plus|mult|num...)) >

This is equivalent to giving a signature to the Prolog terms

assign : Var x Exp $\rightarrow$ Statement

where Var and Exp are types containing the appropriate elements.

However, using types or DTDs it is not possible to express semantic constraints, as for example "The types of the left and right hand sides must be compatible". If the variable $\mathrm{A}$ has been declared as an integer, the statement given as example is not legal.

Web sites (we define a Web sites simply as a set of XML pages) are very similar to programs. In particular, they can be represented as trees, and they may have local constraints expressed by the means of DTDs or XML-shemas. As HTML pages can be translated to XHTML, which is XML with a particular DTD, it is not a restriction to focus only to XML web sites.

There are also differences between Web sites and programs:

- Web sites can be spread along a great number of files. This is the case also for programs, but these files are all located on the same file system. With Web sites we will have to take into account that we may need to access different servers.

- The information is scattered, with a very frequent use of forward references. A forward reference is the fact that an object (or a piece of information) is used before it as been defined or declared. In programs, forward references exist but are most of the time limited to single files so the compiler can compile one file at a time. This is not the case for Web sites and as it is not possible to load a complete site at the same time, we have to use other techniques.

- The syntax can be poorly, not completely or not at all formalized, with some parts using natural languages.

- There is the possibility to use "multimedia". In the word of programs, there is only one type of information to manipulate: text (with structure), so let's say terms. If we want to handle a complete site or document we may want to manipulate images for example to compare the content of an image with a caption. 
- The formal semantics is not imposed by a particular programming language but must be defined by the author or shared between authors as it is already the case for DTDs. This means that to allow the verification of a Web site along its life one will have to define what should be checked.

- We may need to use external resources to define the static semantics (for example one may need to use a thesaurus, ontologies or an image analysis program). In one of our example, we call the wget program to check the validity of URLs in an activity report.

Meta-data are now most of the time expressed using an XML syntax, even if some other concrete syntax can be used (for example the N3 notation [2 can be used as an alternative to the XML syntax of RDF). So, from a syntactic point of view, there is no difference between data and meta-data. Databases also use XML, as a standard interface both for queries and results.

As we can see, the emergence of XML gives us a uniform framework for managing heterogeneous data. Using methods from software engineering will allow a webmaster or an author to check the integrity of its information system and to produce error messages or warnings when necessary, provided that they have made the effort to formalize the semantics rules.

However, this is not yet completely sufficient. Every programmer can relate to some anecdote in which somebody forgets to perform an action as recompiling a part of a program, leading to an incoherent system. Formalizing and mechanizing the management of any number of files is the only way to avoid such misadventure.

Again, at least one solution already exists. It is a utility program named "make" [16], well known to at least unix programmers. This program can manage the dependencies between files and libraries, and it can minimize the actions (such as the calls to the compilers) which must be done when some files have been modified. However, this program can only managed files located on the same file system and must be adapted to handle URLs when a Web site is scattered over multiple physical locations.

\section{EXAMPLES OF SEMANTIC CONSTRAINTS}

For a better understanding of the notion of semantic constraints we will now provide two examples. More examples can be found in the applications section.

- A thematic directory is a site in which documents or external sites are classified. An editorial team is in charge of defining the classification. This classification shows as a tree of topics and subtopics. The intentional semantics of this classification is that a subtopic "makes sense" in the context of the upper topic, and this semantics must be maintained when the site is modified.

To illustrate this, here is an example:

Category: Recreation

Sub-Category: Sports, Travel, Games, Surgery, Music, Cinema

The formal semantics of the thematic directory uses the semantics of words in a natural language. To verify the formal semantics, we need to have access to external resources, maybe a thesaurus, a pre-existing ontology or an ontology which is progressively constructed at the same time as the directory; how to access to this external resource is not of real importance, the important point is that it can be mechanized.

In the former example, the sub-category "Surgery" is obviously a mistake.

- An academic site presents an organization: its structure, its management, its organization chart, etc. Most of the time this information is redundant, maybe even inconsistent. As in a program, one have to identify which part of the site must be trusted: the organization chart or a diary (which is supposed to be up to date) and can be used to verify the information located in other places, in particular when there are modifications in the organization. The "part that can be trusted" can be a formal document such as an ontology, a database or a plain XML document.

The issue of consistency between data and meta-data, or between many redundant data appears in many places, as in the following examples.

- Checking the consistency between a caption and an image; in this case we may want to use linguistic tools to compare the caption with annotations on the image, or to use image recognition tools.

- Comparing different versions of the same site that may exist for accessibility reasons.

- Verifying the consistency between a request to a database, with the result of the request to detect problems in the query and verify the plausibility of an answer (even when a page is generated from a database, it can be useful to perform static verifications, unless the raw data and the generation process can be proved, which is most of the time not the case).

- Verifying that an annotation (maybe in RDF) is still valid when an annotated page is modified.

\section{FORMALIZING WEB SITES}

As seen earlier, Web sites (and other information systems) can be represented as trees, and more precisely by typed terms, exactly as it is the case for programs. It is then natural to apply the formal methods used to define programming languages to Web sites.

In our experiments, we have used the Natural Semantics [ [ 4,12 ] which is an operational semantics derived from the operational semantics of Plotkin [15] and inspired by the sequent calculus of Gentzen [17]. One of the advantages of this semantics is that it is an executable semantics, which means that semantic definitions can be compiled (into Prolog) to generate type-checkers or compilers. Another advantage is that it allows the construction of proofs.

In Natural Semantics, the semantics of programming languages are defined using inference rules and axioms. These rules explain how to demonstrate some properties of "the current point" in the program, named subject, using its subcomponents (i.e., subtrees in the tree representation) and an environment (a set of hypothesis).

To illustrate this, the following rule explains how the statements part of a program depends on the declarative one:

$$
\frac{\emptyset \vdash \text { Decls } \rightarrow \rho \quad \rho \vdash \text { Stmts }}{\vdash \text { declare Decls in Stmts }}
$$

The declarations are analyzed in an empty environment $\emptyset$, constructing $\rho$ which is a mapping from variable names to declared 
types. This environment is then used to check the statements part, and we can see that in the selected example the statements part do not alter this environment.

These inference rules can be read in two different ways: if the upper part of the rule has been proved, then we can deduce that the lower part holds; but also in a more operational mode, if we want to prove the lower part we have to prove that the upper part holds.

The following rule is an axiom. It explains the fact that in order to attribute a type to a variable, one has to access the environment.

$$
\rho \vdash \operatorname{var} X: T \quad\{X: T\} \in \rho
$$

"Executing" a semantic definition means that we want to build a proof tree, piling up semantic rules which have been instantiated with the initial data.

First, we have made experiments using directly Natural Semantics [\$, 6]. These experiments showed that this style of formal semantics perfectly fits our needs, but it is very heavy for end-users (authors or webmaster) in the context of Web sites. Indeed, as we can see in the former rules, the recursion is explicit, so the specification needs at least one rule for each syntactical operator (i.e., for each XML tag). Furthermore, managing the environment can be tedious, and this is mainly due to the forward declarations, frequent in Web sites (Forward declarations means that objects can be used before they have been defined. This implies that the verifications must be delayed using an appropriate mechanism as coroutine, or using a two-pass process).

It is not reasonable to ask authors or webmasters to write Natural Semantics rules. Even if it seems appropriate for semantic checking, the rules may seem too obscure to most of them. Our strategy now is to specify a simple and specialized specification language that will be compiled in Natural Semantics rules, or more exactly to some Prolog code very close to what is compiled from Natural Semantics.

We can make a list of requirements for this specification language:

- No explicit recursion.

- Minimizing the specification to the points of interest only.

- Simple management of the environment.

- Allowing rules composition (points of view management).

- Automatic management of forward declarations.

In a second step we have written various prototypes directly in Prolog. The choice of Prolog comes from the fact that it is the language used for the implementation of Natural Semantics. But it is indeed very well adapted to our needs: terms are the basic objects, there is pattern matching and unification.

After these experiments, we are now designing specification language. Here are some of the main features of this language:

- Patterns describe occurrences in the XML tree. We have extended the language XML with logical variables. In the following examples variable names begin with the \$ sign.

- A local environment contains a mapping from names to values. The execution of some rules may depend on these values. A variable can be read $(=)$ or assigned $(:=)$.

- A global environment contains predicates which are assertions deduced when rules are executed. The syntax of these predicates is the Prolog syntax, but logical variables are marked with the $\$$ sign. The sign $=>$ means that its right hand side must be added to the global environment.
- Tests enable us to generate warnings or error messages when some conditions do not hold. These tests are just now simple Prolog predicates. The expression ? pred / error means that if the predicate pred is false, the error message must be issued. The expression ? pred $->$ error means that if the predicate pred is true, the error message must be issued.

Semantic rules contain two parts: the first part explains when the rule can be applied, using patterns and tests on the local environment; the second part describes actions which must be executed when the rule applies: modifying the local environment (assignment), adding of a predicate to the global environment, generating a test.

Recursion is implicit. It is also the case for the propagation of the two environments and of error messages.

In the following section, we present this language with more details.

\section{A SPECIFICATION LANGUAGE TO DEFINE THE SEMANTICS OF WEB SITES}

We give in this section a complete description of our specification language. The formal syntax is described in Appendix A.

\subsection{Patterns}

As the domain of our specification language is XML document, XML elements are the basic data of the language. To allow patternmatching, logical variables have been added to the XML language (which is quite different from what is done in XSLT).

Variables have a name prefixed by the \$ sign, for example: \$X. Their also exists anonymous variables which can be used when a variable appears only once in a rule: \$_. For syntactical reasons, if the variable appears in place of an element, it should be encapsulated between $<$ and $\rangle:\langle \$ \mathrm{X}\rangle$.

In a list, there is the traditional problem to know if a variable matches an element or a sublist. If a list of variables matches a list of elements only the last variable matches a sublist. So in

$<$ tag $><\$ A><B></$ tag $>$

the variable $\$ A$ matches the first element contained in the body of $<$ tag $>$ and \$B matched the rest of the list (which may be an empty list).

When a pattern contains attributes, the order of the attributes is not significant. The pattern matches elements that contain at least the attributes present in the pattern.

For example, the pattern

$<$ citation year $=\$ \mathrm{Y}>\langle \$ \mathrm{~T}>\langle\mathbf{R}></$ citation $>$

matches the element

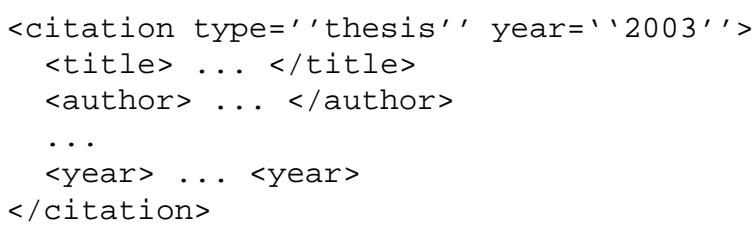

binding \$Y with " 2003 "

and $\$ \mathrm{~T}$ with $\langle$ title $>\ldots</$ title $>$. The variable $\$ \mathrm{R}$ is bound to the rest of the list of elements contained in <citation $\rangle$, i.e., the list of elements 


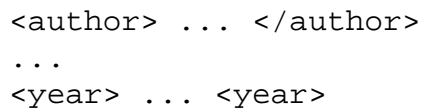

When this structure is not sufficient to express some configuration (typically when the pattern is too big or the order of some elements is not fixed), one can use the following "contains" predicate:

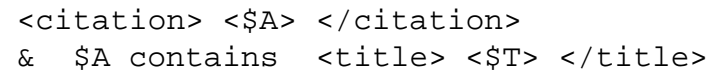

In this case the element $\langle$ title $\rangle$ is searched everywhere in the subtree \$A.

\subsection{Rules}

A rule is a couple containing at least a pattern and an action. The execution of a rule may be constrained by some conditions. These conditions are tests on values which have been computed before and are inherited from the context. This is again different from XSLT in which it is possible to get access to values appearing between the top of the tree and the current point. Here these values must explicitly be stored and can be the result of a calculus.

In the following rule, the variable $\$ \mathrm{~A}$ is bound to the year of publishing found in the <citation> element while $\$ \mathrm{~T}$ is found in the context.

$<$ citation year $=\$ Y><$ P $></$ citation $>$

$\&$ currentyear $=\$ \mathrm{~T}$

The effect of a rule can be to modify the context (the modification is local to the concerned subtree), to assert some predicates (this is global to all the site) and to emit error messages depending on tests.

The following example sets the value of the current year to what is found in the argument of the <activityreport> element.

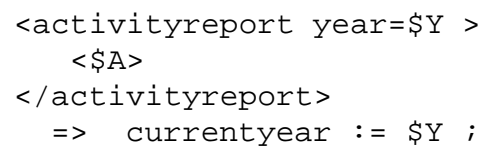

The two following rules illustrate the checking of an untrusted part of a document against a trusted part.

In the trusted part, the members of a team are listed (or declared). The context contains the name of the current team, and for each person we can assert that it is a member of the team.

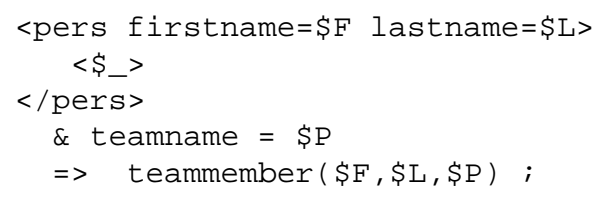

In the untrusted part, we want to check that the authors of some documents are declared as members of the current team. If it is not the case, an error message is produced.

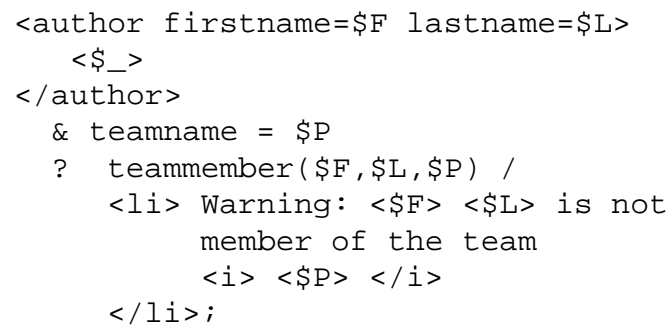

\subsection{Dynamic semantics}

This section explains how semantic rules are executed.

On each file, the XML tree is visited recursively. A local environment which is initially empty is constructed. On each node, a list of rules which can be applied (the pattern matches the current element and conditions are evaluated to true) is constructed, then applied. This means that all rules which can be applied are evaluated in the same environment. The order in which the rules are then applied is not defined.

During this process, two results are constructed. The fist one is a list of global assertions, the second is a list of tests and related actions. These are saved in files.

A global environment is constructed by collecting all assertions coming from each different environment files. Then using this global environment, tests are performed, producing error messages if there are some.

To produce complete error messages, two predefined variables exist: \$SourceFile and \$SourceLine. They contain respectively, the name of the current file which is analyzed and the line number corresponding to the current point. This is possibly due to the use of a home made XML parser which is roughly described in Appendix A.

\section{APPLICATIONS}

\subsection{Verifying a Web site}

The following example illustrates our definition language. We want to maintain an academic site. The current page must be trusted and contains a presentation of the structure of an organization.

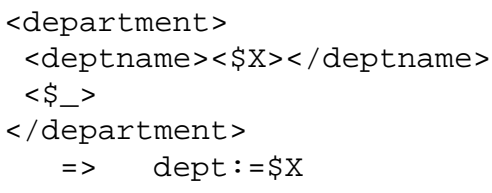

The left hand side of the rule is a pattern. Each time the pattern is found, the local environment is modified: the value matched by the logical variable $\$ \mathrm{X}$ is assigned to the variable dept. This value can be retrieved in all the subtrees of the current tree, as in the following example. \$_ matches the rest of what is in the department tag.

Notice that, unlike what happens in XSLT in which it is possible to have direct access to data appearing between the root and the current point in a tree, we have to store explicitly useful values in the local environment. In return one can store (and retrieve) values which are computed and are not part of the original data, and this is not possible with XSLT.

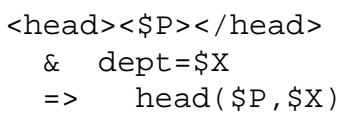

We are in the context of the department named $\$ \mathrm{X}$, and $\$ \mathrm{P}$ is the head of this department. head ( $\$ \mathrm{P}, \$ \mathrm{X})$ is an assertion which is true for the whole site, and thus we can add this assertion to the global environment. This is quite equivalent to building a local ontology. If, in some context, an ontology already exists, we can think of using it as an external resource. Notice that a triplet in RDF can be viewed as a Prolog predicate [14].

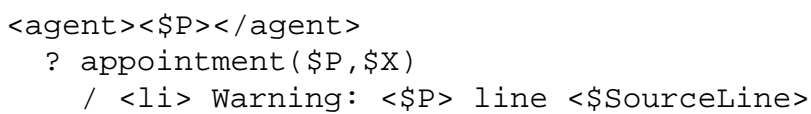




\section{does not appear in the current \\ staff chart. \\ $</ l i>$;}

This rule illustrates the generation of error messages. In the XML text, the tag agent is used to indicate that we have to check that the designated person has got an appointment in a department. The treatment of errors hides some particular techniques as the generated code must be rich enough to enable to locate the error in the source code.

\subsection{Verifying a document and inferring new data}

As a real sized test application, we have used the scientific part of the activity reports published by Inria for the years 2001 and 2002 which can be found at the following URLs: http://www.inria.fr/rapportsactivite/RA2001/index.html and http://www.inria.fr/rapportsactivite/RA2002/index.html.

The sources of these activity reports are LaTex documents, and are automatically translated into XML to be published on the Web.

The XML versions of these documents contain respectively 108 files and 125 files, a total of 215000 and 240000 lines, more than 12.9 and 15.2 Mbytes of data. Each file is the reflect of the activity of a research group. Even if a large part of the document is written in French, the structure and some parts of the document are formalized. This includes parts speaking of the people and the bibliography.

The source file of our definition can be found in Appendix B.

Concerning the people, we can check that names which appears in the body of the document are "declared" in the group members list at the beginning. If it is not the case, the following error message is produced:

Warning: $\mathrm{X}$ does not appear in the list of project's members (line $\mathrm{N}$ )

Concerning the bibliography of the group, the part called "publications of the year" may produce error messages like the following one:

Warning: The citation line 2176 has not been published during this year (2000)

We have also use Wget to check the validity of URLs used as citation, producing the following error messages:

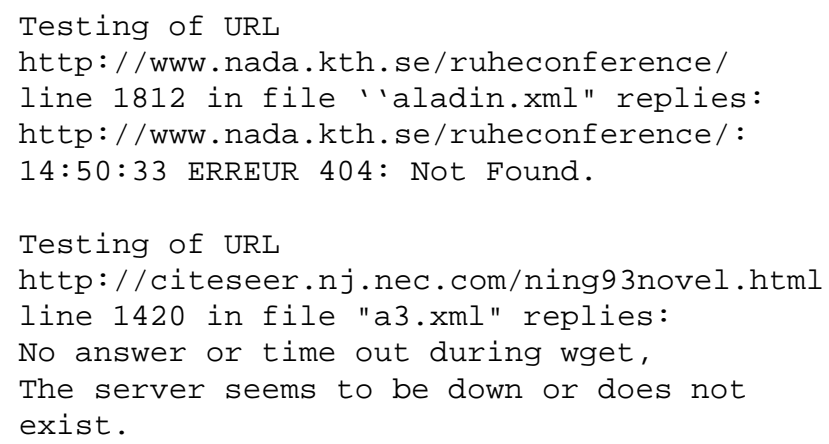

Beyond these verification steps, using a logic programming approach allows us to infer some important information. For example we found out that 40 publications out of a total of 2816 were co-written by two groups, giving an indicator on the way the 100 research groups cooperate.
The citation "Three knowledge representation formalisms for content-based manipulation of documents" line 2724 in file "acacia.xml" has been published in cooperation with

orpailleur.

Our system reported respectively 1372 and 1432 messages for the years 2001 and 2002. The reasons of this important number of errors are various. There is a lot of misspelling in family names (mainly due to missing accents or to differences between the name used in publications and the civil name for women). Some persons who participated to some parts of a project but have not been present during all the year can be missing in the list of the team's members. There is also a lot of mistakes in the list of publications: a paper written during the current year can be published the year after and should not appear as a publication of the year. There are also a lot of errors in URLs and this number of errors should increase as URLs are not permanent objects.

It is to be noticed that we have worked on documents that have been generated, and that the original latex versions have been carefully reviewed by several persons. This means that proofreading is not feasible for large documents.

For future developments, other important indicators can also be inferred: how many $\mathrm{PhD}$ students are working on the different teams, how many $\mathrm{PhD}$ theses are published, etc. These indicators are already used by the management to evaluate the global performance of the institute but are compiled manually. An automatic process should raise the level of confidence in these indicators. They can also be compared mechanically with other sources of information as, for example, the data bases used by the staff office.

\section{IMPLEMENTATION NOTES}

All the implementation has been done in Prolog (more exactly Eclipse) except the XML scanner which has been constructed with flex.

An XML parser has been generated using an extension of the standard DCG. This extension, not yet published, gives some new facilities as allowing left recursive rules and generating some efficient prolog code. A particularity is that it constructs two resulting terms. The first one is a parsed term, as usual. The second one is used to allow a correspondence between an occurrence in the parsed term and the line numbers in the source, allowing pertinent error messages as seen in the previous section.

This parser has been extended to generate our specification language parser.

The rule compiler as been entirely written in Prolog.

Concerning the execution of the specification, the main difficulty comes from the fact that we have a global environment. The traditional solution in this case is to use coroutines or delays. As our input comes from many files, this solution was not reasonable, and we have chosen a two pass process. For each input file, during the first pass we use the local environment and construct the part of the global environment which is generated by the current file and a list of delayed conditions which will be solved during the second pass. During the second pass, all the individual parts of the global environment are merged and the result is used to perform the delayed verifications, producing errors messages when necessary.

\section{CONCLUSION}

In this paper, we have showed how techniques used to define the formal semantics of programming languages can be used in the context of Web sites. This work can be viewed as a complement 
to other researches which may be very close: for example in [9], some logic programming methods are used to specify and check integrity constraints in the structure of Web sites (but not to its static semantics). Our work which is focused on the content of Web sites and on their formal semantics, remains original. It can be extended to the content management of more general information system.

As we have seen, the techniques already exist and are used in some other domains. The use of logic programming, and in particular of Prolog as an implementation language, is very well adapted to our goals. Our goal is to make these techniques accessible and easily usable in the context of Web sites with the help of a specific specification language.

We are now convinced that our technology is adequate. We plan, in parallel with the development of our language, to explore more deeply some applications, both on the verification side and the inference side.

\section{ACKNOWLEDGMENT}

The author wants to thank Brigitte Trousse for its participation in an earlier stage of this work.

\section{REFERENCES}

[1] T. Berners-Lee. A Road Map to the Semantic Web, September 1998. W3C http://www.w3.org/DesignIssues/Semantic.html.

[2] T. Berners-Lee. Ideas about web architecture - yet another notation notation 3, 2001. W3C http://www.w3.org/DesignIssues/Notation3.html.

[3] T. Berners-Lee, J. Hendler, and O. Lassila. The semantic web. In Scientific American, May 2001.

[4] T. Despeyroux. Executable Specification of Static Semantics. In Semantics of Data Types, Lecture Notes in Computer Science, Vol. 173, June 1987.

[5] T. Despeyroux and B. Trousse. Semantic verification of web sites using natural semantics. In RIAO 2000, 6th Conference on "Content-Based Multimedia Information Access", College de France, Paris, France, April 2000.

[6] T. Despeyroux and B. Trousse. Maintaining semantic constraints in web sites. In AACE WebNet 2001 Conference, Orlando, Florida, October 2001.

[7] D. Fensel, J. Angele, S. Decker, M. Erdmann, H.-P. Schnurr, R. Studer, and A. Witt. On2broker: Lessons Learned from Applying AI to the Web. Technical report, Institute AIFB, 1998.

[8] D. Fensel, R. Decker, M. Erdman, and R. Studer. Ontobroker: the Very High Idea. In Proceedings of the 11th International FLAIRS Conference (FLAIRS-98), May 1998.

[9] M. F. Fernandez, D. Florescu, A. Y. Levy, and D. Suciu. Verifying integrity constraints on web sites. In IJCAI, pages 614-619, 1999.

[10] C. A. Gunter. Semantics of Programming Languages. MIT Press, 1992.

[11] H. Hosoya and B. Pierce. Xduce: A typed xml processing language. In Proceedings of Third International Workshop on the Web and Databases, May 2000.

[12] G. Kahn. Natural Semantics. In Proceedings of the Symp. on Theorical Aspects of Computer Science, TACS, Passau, Germany, 1987. LNCS 247, Springer-Verlag, Berlin. also Inria Research Report 601, February 1987.

[13] E. Meijer and M. Shields. $\mathrm{XM} \lambda$ : A functional programming language for constructing and manipulating $\mathrm{xml}$ document, 1999. Draft, http://www.cse.ogi.edu/ mbs/pub/xmlambda/.
[14] J. Peer. A logic programming approach to RDF document and query transformation. In Workshop on Knowledge Transformation for the Semantic Web at the 15th European Conference on Artificial Intelligence, Lyon, France, 2002.

[15] G. D. Plotkin. A structural approach to operational semantics. Technical Report DAIMI FN-19, Aarhus University, 1981.

[16] R. M. Stallman and R. McGrath. GNU Make: A Program for Directing Recompilation, for Version 3.79. Free Software Foundation, 675 Mass Ave., Cambridge, MA 02139, USA, Tel: (617) 876-3296, USA, 2000.

[17] E. Szabo. The Collected Papers of Gerhard Gentzen. North-Holland, Amsterdam, 1969.

[18] F. van Harmelen and D. Fensel. Practical Knowledge Representation for the Web. In D. Fensel, editor, Proceedings of the IJCAI'99 Workshop on Intelligent Information Integration, 1999.

[19] F. van Harmelen and J. van der Meer. Webmaster: Knowledge-based Verification of Web-pages. In Twelfth International Conference on Industrial and Engineering Applications of Artificial Intelligence and Expert Systems IEA/AIE'99, 1999.

[20] W3C. Xml, xsl, xml schema and rdf recommendations or submissions. W3C http://www.w3.org/.

[21] W3C. Daml+oil (march 2001) reference description, 2001. W3C http://www.w3.org/TR/daml+oil-reference.

\section{APPENDIX}

\section{A. OUR SPECIFICATION LANGUAGE SYNTAX}

This appendix contains the source file of our parser. It uses a home made extension of Define Clause Grammars. This extension has three advantages: it handles left recursions; it takes advantage of prolog hash-coding over some arguments; it produces a structure which is rich enough to allow precise error messages with some back references to the source that is analysed (it can be used in particular to retrieve line numbers).

The production

$\operatorname{attr}(\operatorname{attr}(\mathrm{N}, \mathrm{V}))$ : $-\operatorname{name}(\mathrm{N}),\left[{ }^{\prime}={ }^{\prime}\right], \operatorname{value}(\mathrm{V})$. is equivalent to the traditional grammar rule

attr $->$ name ' $={ }^{\prime}$ value.

$\operatorname{attr}(\mathrm{N}, \mathrm{V})$ is a regular prolog term and explains how the parse tree is constructed from subtrees.

The call to stoken is the interface with the lexer which is implemented using flex.

Beside the specification language itself, we can recognize the syntax of prolog terms (non terminal term) and XML elements (non terminal element). Traditional elements are extended with variables as in $<$ \$varname $>$. The sign $<\star$ is used to comment easily some complete rules.

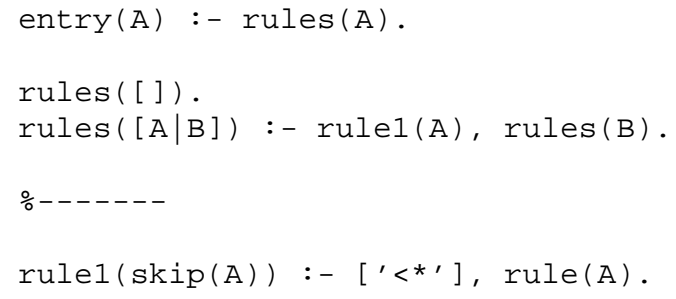




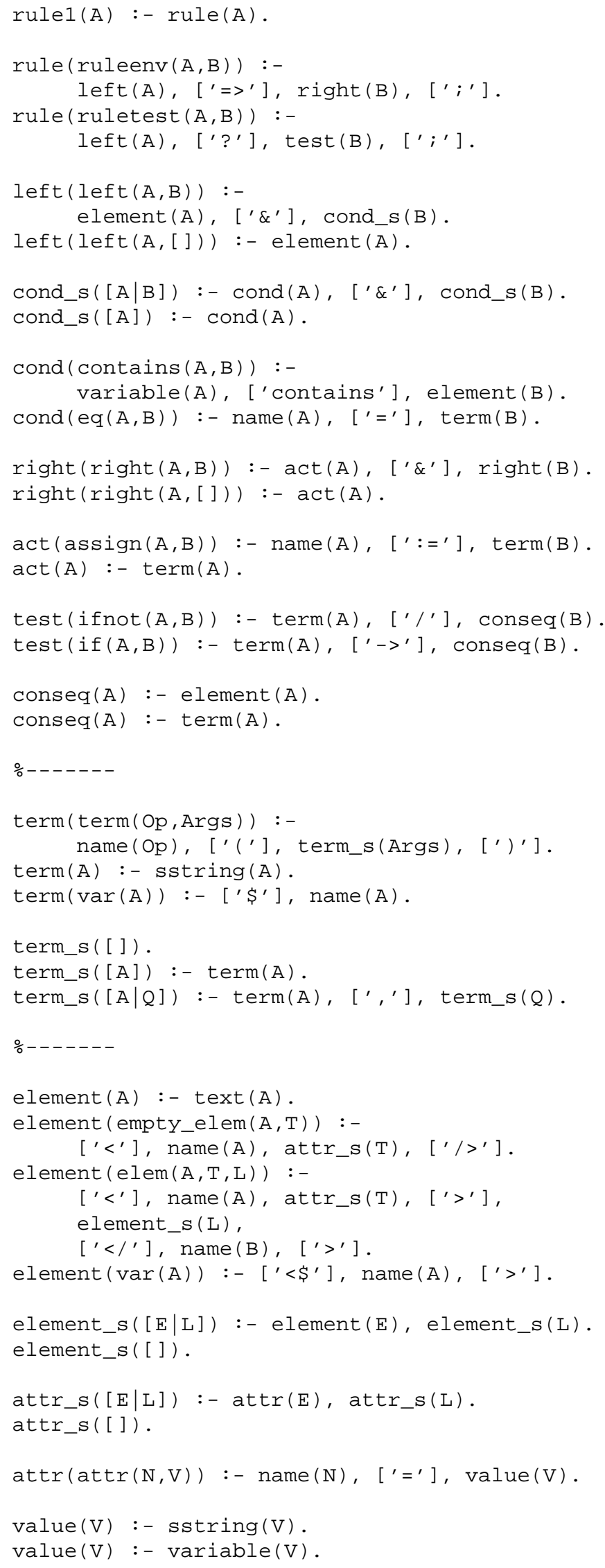

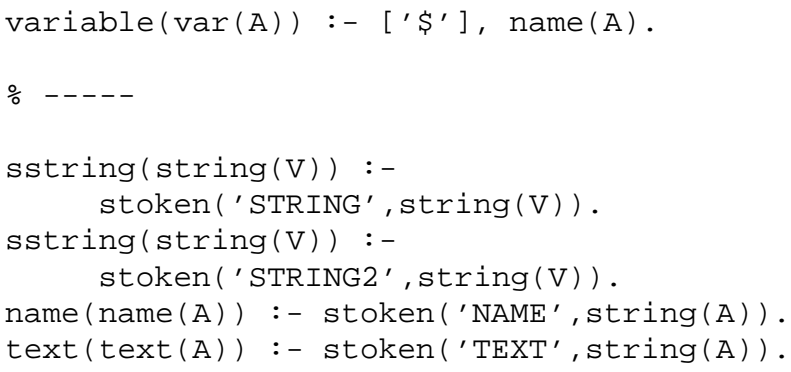

\section{B. SOURCE FOR CHECKING SOME PART OF AN ACTIVITY REPORT}

This appendix contains the source file for our activity report checker. This is the real source, and it may differ from what appears in the text.

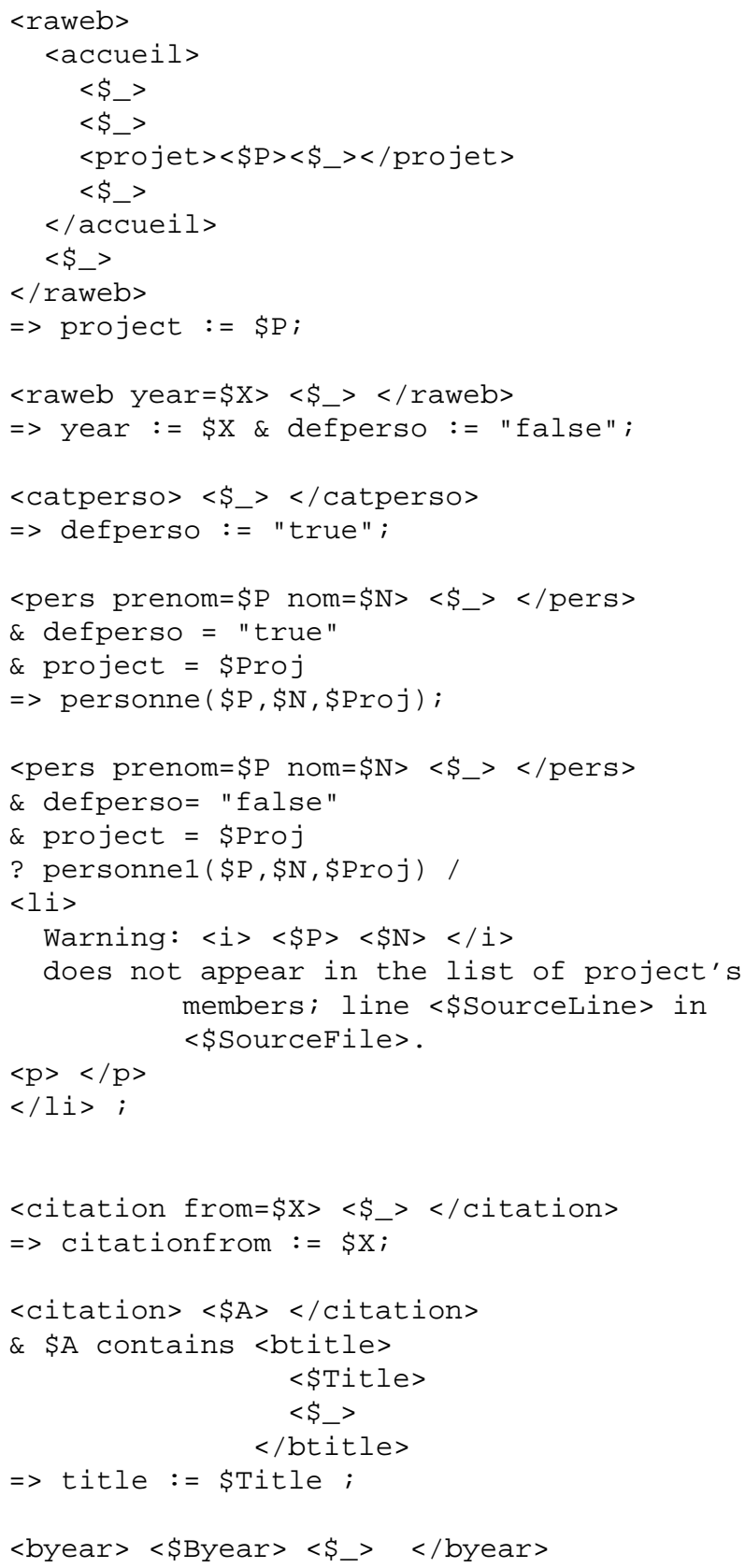




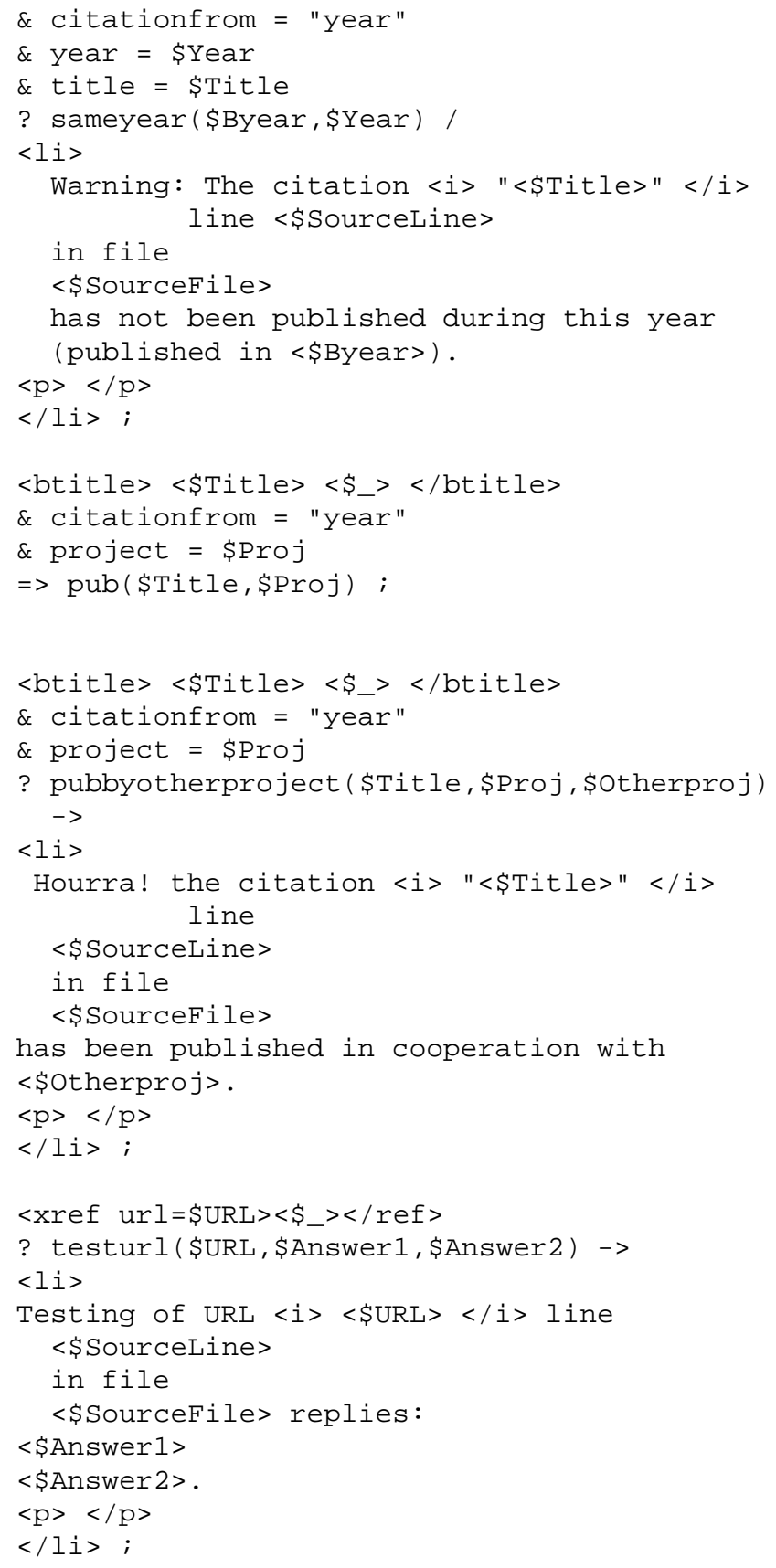

The predicates personne1, sameyear, pubbyotherproject, and testurl are defined directly in Prolog. The last one makes a call to the program wget with some timeout guard. 